\title{
CAMPI ELETTROMAGNETICI VORTICOSI IN LASTRE INFINITAMENTE ESTESE E DI DIMENSIONI FINITE
}

\author{
A. Belluigi
}

Nel 1949 accennai $\left({ }^{1}\right)$ ad una soluzione del problema dell'induziono e.m. relativa ad una "lastra " o strato conduttivo sottile, di dimensioni finite (a contorno rettangolare), perpendicolare alla superficie orizzontale del suolo con emittore percorso da c.a. a bassa frequenza, tale che la densità dei c.m.p. risulti perpendicolare allo strato.

Sviluppai successivamente, per esteso $\left({ }^{2}\right)$, la teoria, e la soluzione del problema venne ricondotta a quella d'una equazione integrale di $2^{n}$ specie integrabile con delle limitazioni sul valore di alcuni rapporti di grandezze fisico-geometriche, senza ripercussioni per questo sulla natura del problema posto.

La lastra è l'idealizzazione ad es. di un giacimento minerario conduttivo, (vena o specchio minerale), di tipo bidimensionale, a seconda il rapporto relativo delle sue dimensioni geometriche, due delle quali, in ogni caso, sempre prevalenti sullo spessore, o "potenza " di giacimento.

In una terza Nota $\left({ }^{3}\right)$ svolsi particolarmente alcune considerazioni applicative, con la descrizione di nuove necessarie apparecchiature e.m. tribobiniche (Mind, Trib), tendenti ad eliminare, nelle misure, il c.e.m.p. (primario), nelle condizioni della sua alta prevalenza sul c.e.m.s. (secondario), altro problema fondamentale dalla cui soluzione spesso dipende il successo o meno dei metodi elettroinduttivi.

Nell'impostazione teorica (2) assunsi, quale induttore, uno chiuso tale che il vettore densità di c.m. risultasse perpendicolare alla lastra, nel suo centro geometrico, preferibilmente, senza ulteriori specificazioni. In realtà tale emittore si può agevolmente realizzare con una bobina circolare poliassiale (emittrice del Trib.). $\mathbf{E}$ da tener presente che solo la componente della densità di flusso m. normale alla striscia può attivare correnti vorticose nellipotesi fatta della limitazione della potenza dello strato rispetto alle altre due sue dimensioni.

In prossimità della bobina inducente (3), (ad es. nella sua posizione verticale), e nello stesso piano, si colloca una $2^{\text {a }}$ bobina (scher- 
mante), mentre il quadro ricevente, sempre complanare (quadri a contorni associati) si pone a fianco dello schermante, dalla parte più lontana dell'emittore. Ne risulta un complesso tribobinico asimmetrico che chiamai Trib, atto alla misura dei soli c.e.m.s., quando il quadro intermedio schermante, inserito in apposito circuito di compensazione, con capacità manovrabile, è atto a compensare, in grandezza e fase, il c.m. primario nell'ambito del quadro ricevente, che rimane perciò nel. l'ombra e.m. del c.e.m.p.

Contemporaneamente al ciclo di lavori, $\left({ }^{1}\right),\left({ }^{2}\right),\left({ }^{3}\right)$, (occupandomi in quel periodo soprattutto di rilevamenti geoelettrici e.m. in galleria di miniere di solfuri misti (B. G.) in Sardegna), esposi in (4) i risultati fondamentali di altri miei studi sulle leggi dei c.e.m.s. nelle lastre conduttrici, orizzontali, ma $\infty^{\text {te }}$ estese, considerando un emittore circolare (alimentato da c.a.), integrando classici indirizzi teorici vertenti sull'argomento tra cui quelli di Max Abraham e Anton Graf.

I problemi risolti in $\left(^{4}\right)$ differiscono sostanzialmente, da un punto di vista strettamente analitico, da quelli risolti in $\left({ }^{2}\right)$, pur trattandosi dell'esame di c.e.m. vorticosi provocati con gli stessi induttori chiusi, bobinici, ad avvolgimenti piano-paralleli agli indotti lastriformi, (indotti estesi o di dimensini finite).

Ma se la differenza analitica dell'impostazione dei due problemi è sostanziale, non lo è affatto la differenza delle soluzioni, a significato fisico-geologico, l' $\infty^{\text {te }}$ esteso matematico riducendosi in realtà ad un "molto esteso", per cui le soluzioni non potranno in linea di massima differire granche tra di loro com'è intuibile, e come mi propongo di evidenziare qui.

Tuttavia differenze (inessenziali da un certo punto di vista) si rileveranno anzitutto in relazione alla peculiarità stessa dei problemi, (per cui si dovranno risentire le caratteristiche dimensionali degli indotti nei 2 casi), secondariamente in conseguenza delle modalità d'energizzazione, dei tipi di emittori, chiusi o aperti, della loro posizione relativa rispetto ai giacimenti, dell'orientamento stesso dei giacimenti lastriformi rispetto alla superficie del suolo (ritenuta orizzontale) ove si effettuano le misure.

L'induzione e.m., con emittore circolare orizzontale, in una lastra orizzontale $\infty^{\text {te }}$ estesa, produce in essa correnti vorticose orizzontali circolari concentriche, mentre in una lastra $\infty^{\text {te }}$ estesa o di dimensioni finite ma verticale, le correnti vorticose permarranno in un piano verticale, con effetti e.m. distinti, nei 2 casi, alla superficie del suolo. 
Emittori aperti rettilinei $\propto^{\text {te }}$ estesi, provocano correnti indotte parallele, di forme e giacitura spaziale perciò distinte da quelle dovute ad emittori chiusi, con conseguenti ripercussioni sui c.e.m.s. che si misurano nella prospezione di superficie.

Il c.e.m.s. relativo alla lastra conduttiva sottile, orizzontale, este$\mathbf{s a},\left({ }^{4}\right)$, di potenza " $\mathbf{d}$ ) (o più generalmente a " piccolo fattore d'induzione ' $\mathrm{f}$ ' $)$, provocato da un emittore circolare di raggio $a$, attra-

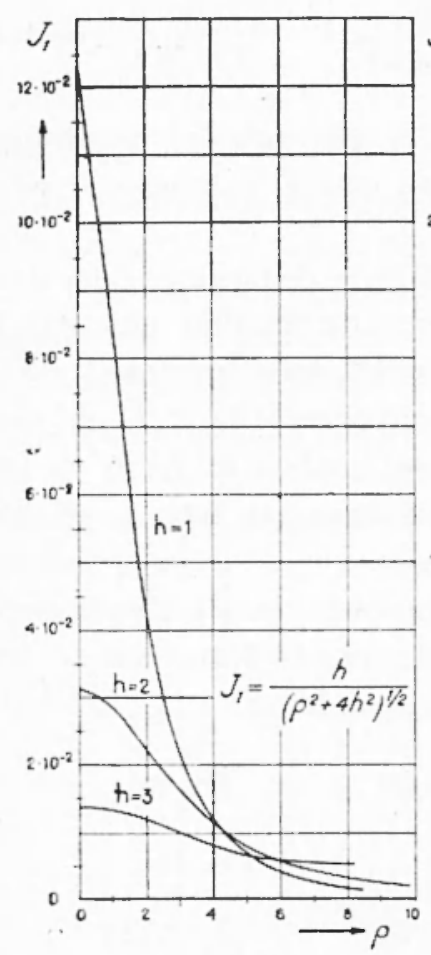

Fig. 1

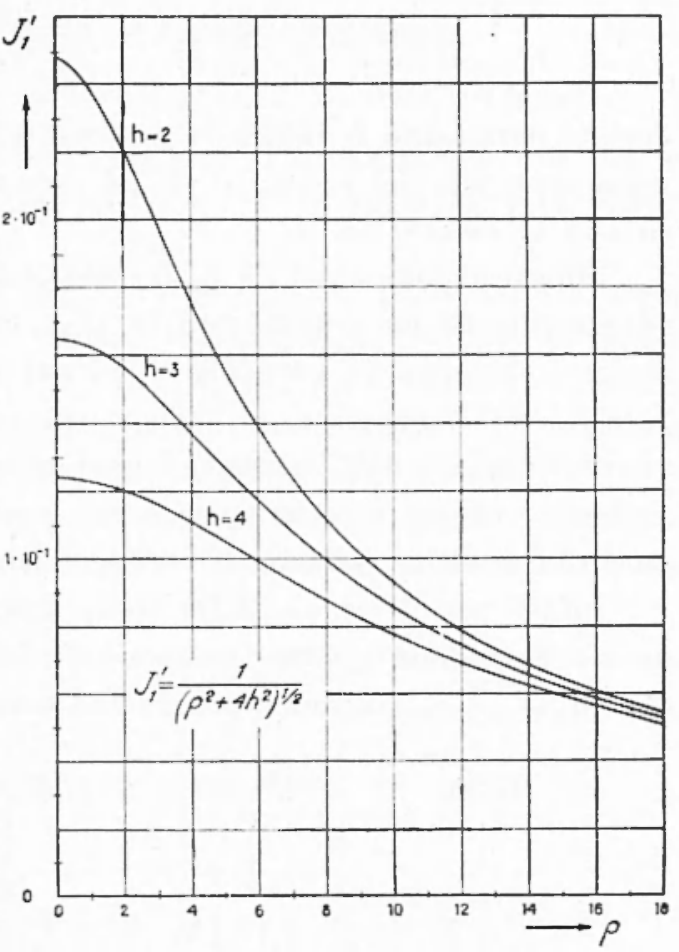

Fig. 2

versato da cai. con $J$ amperè-spire, parallelo, alla lastra stessa, appoggiato sul terreno, ha componenti (radiali e verticali) cosi esprimibili (tenendo conto degli sfasamenti rispetto ad $J$ ):

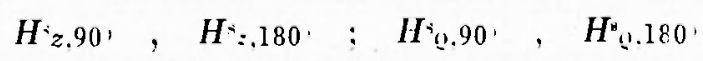

$\Lambda$ rigore se il "fattore d'induzione" rientra in determinati limiti, $\left(f=\gamma \cdot \mu . \sigma . a . d . \leqslant \lambda\right.$, con usuale significato $-v \cdot\left({ }^{4}\right)-$ dei simboli e grandezze), l'attributo di "piccolo fattore d'induzione", pertinente alla lastra, può comportare sia la considerazione di lastre sottili, che relativamente spesse, purché valga la disugruaglianza che caratterizza " $f$ ". 
Tali grandezze e.m. sono proporzionali ad altre quattro da cui ne derivano l'andamento spaziale istantaneo: $J_{1}, J_{1}^{\prime}, J_{2 .}, J_{2}$ (primi termini di particolari sviluppi in serie delle funzioni $\boldsymbol{H}_{*}$ di $\mathrm{M}$. Abraham).

Vale la seguente corrispondenza di proporzionalita:

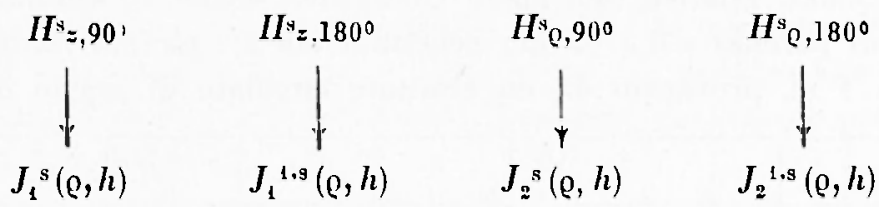

dove il paramento $h$ indica la profondità di giacitura del conduttore dalla superficie del suolo, $(s)$ sta ad indicare che le grandezze si riferiscono al c.e.m.s. (v. 4).

Siffatti andamenti delle $J_{1}$, in funzione della distanza $\varrho$, sono stati estesamente da me commentati in $\left({ }^{4}\right)$, per varie finalità; qui per̀̀ $\grave{e}$ da " evidenziare ", in particolare, le similitudini delle funzioni $J_{1}$ e $J_{1}$; $J_{2}$ e $J_{2}^{\prime}$; la $1^{\text {a }}$ coppia $J_{1}, J_{1}^{\prime}$ con massimi all'origine, la $2^{\mathrm{a}} J_{: 丷,} J_{2}^{\prime}$ con massimi discosti dall'origine nel qual punto i valori di $J_{2}, J_{2}^{\prime}$ si annullano, "coppie caratteristiche" che permangono in tutti i casi, sia pure con qualche variante di corrispondenza.

Infatti per il c.e.m.s. della lastra finita verticale (2), l'espressioni da me date delle quattro componenti del c.m., indicate con le lettere $M, N, P, Q$, seguono la seguente corrispondenza:

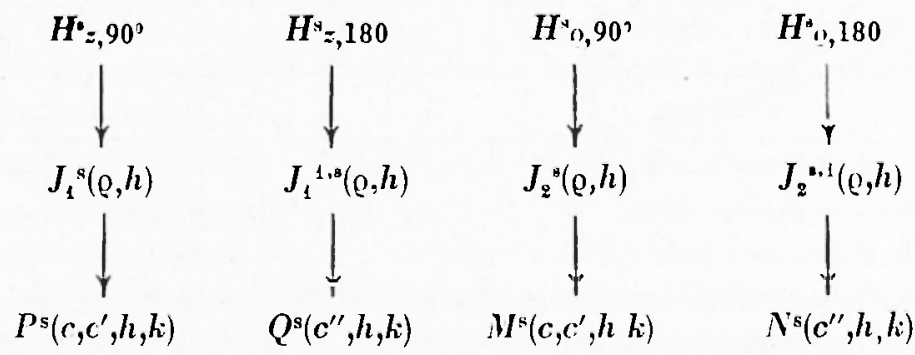

Riportai in (2), per brevità, solo i grafici di $N$ e $Q$ in funzione di: $\frac{x}{l} \quad i=$ semiampiezza verticale della lastra di piccolo spessore: $w<<2 l<<2 L ;(2 l, 2 L)=$ contorno rettangolare; $x=$ ascissa perperpendicolare alla lastra nel suo centro di figura, origine degli assi di simmetria $(x, y, z)$; parametro variabile: $k=z / l$.

Il piano orizzontale della superficie del suolo, parallelo al piano 
$x y$ del riferimento, è tale che: $z \geqslant l:$ (condizione ciò̀ d'interramento o di affioramento dello strato).

È superfluo trascrivere - v. $\left(^{(2)}-\right.$ l'espressioni analitiche delle funzioni $M, N, P, Q$, solo ricordo che esse dipendono da $h, k$, ( $h$ qui è un rapporto fra 2 lunghezze: $\frac{x}{l}=h$ con significato correlazionabile alle " $\varrho$ " delle $J_{1}, k=\frac{z}{l}$ pure è un rapporto fra 2 lunghezze correlazionahili con le " $h$ ) delle $J_{1}$ ), e dai tre coefficienti:

$$
c=c(l, b,(1), s), c^{\prime}=c^{\prime}\left(l, b,(1, s), c^{\prime \prime}=c^{\prime \prime}(l, b,(1), s)\right.
$$

dove: " $b$ " è l'ampiezza massima del c.m. alternato impresso $B_{\mathrm{i}}$, ( (i) ") è la pulsazione della c.a. agente, “ $s$ » è lo spessore pellicolare: $s=\left(u \sigma(1)^{-1 / 2}\right.$ del conduttore.

I profili dei coefficienti $M, N, P, Q$, tracciati numerosi per scopi applicativi, in situazioni di diverse semiampiezze verticali di strato convenientemente scelto: $(l=1,3,5,7,10)$, e di diverse profondità $(k=2,3,5,7,10)$, con le ascisse in scale logaritmiche $(x / l$ variabile da 0,1 a 10 , e le ordinate in scala lineare, o con entrambe le coordinate in scale logaritmiche, o in scale lineari, (a seconda la convenienza pratical, seguono in un "Album" con un centinaio di curve, e migliaia di punti calcolati.

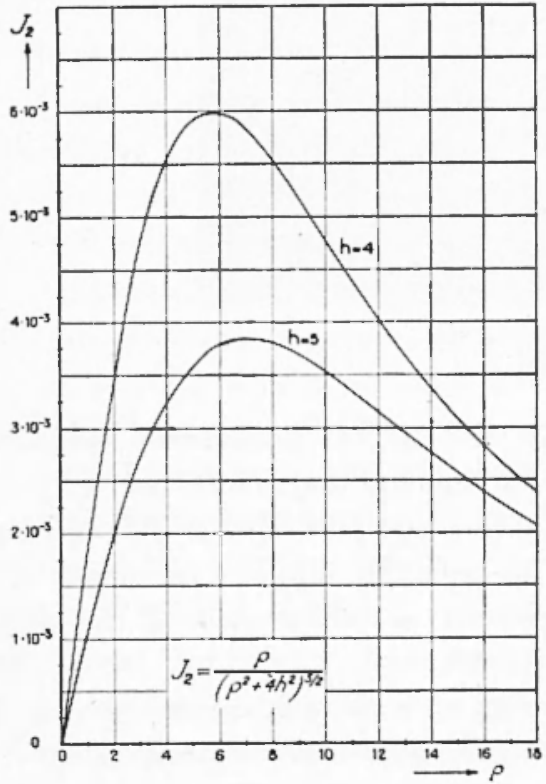

Fig. 3

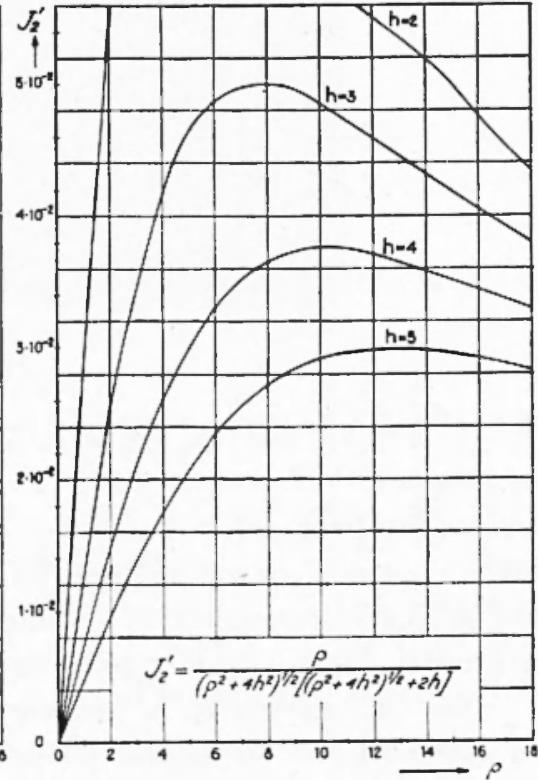

Fig. 4 
Ma ai fini semplicemente dimostrativi è sufficiente considerare quattro diagrammi caratteristici di essi ( $P, Q, M, N$, vedi "Album ) in Appendice) per il paragone con i quattro delle Fig. 1, 2, 3, 4 surriportati (tratte dalla pubblicazione del 1948, nella quale appare altresi una estesa bibliografia).

Analizzando intanto l'andamento di un coefficiente ad es. $M$

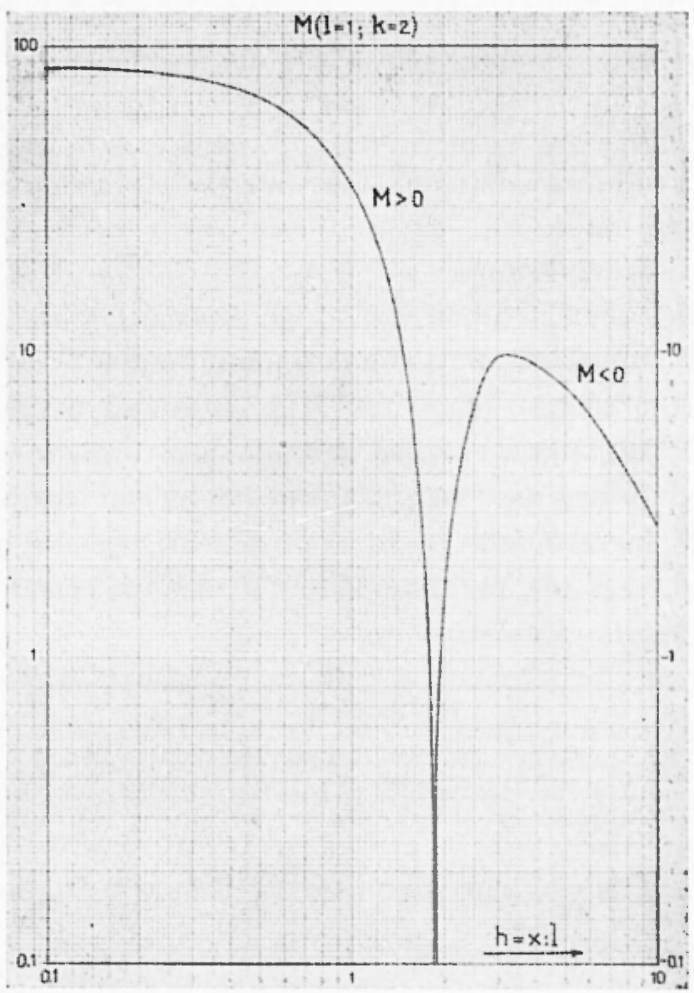

Fig. 5

$(l=1, k=2$ ), osservo, (v. Fig. 5), che $M>O$ diminuisce con l'aumentare di: $x / l$, mentre $M<O$ s'incrementa dapprima per poi tendere assintoticamente all'ascissa: (questo comportamento caratteristico delle "lastre ", essenzialmente bidimensionali, appare in modo incisivo in scala bilogaritmica, e differenzia in modo netto il tipo bidimensionale esteso $J$, da quello finito $M$ ).

Nei vari profili $J_{1}$, in genere, non si nota mai, qualunque sia $P$, un cambiamento di segno, proprietà questa che caratterizza invece le $M, N$. 
Come si può osservare, con ampia casistica, nell'annesso Album, per una data " $l$ " aumentando: $h=x / l, M>O$ diminuisce più o meno rapidamente, (a seconda il valore minore o maggiore $d i k$ ), salvo passare, ad un certo punto, a valori: $M<O$ con caratteristico comportamento di minimo. Inoltre $h=x / l$ può aumentare con $x$, ossia con l'allontanarsi dell'osservatore dalla lastra, o col diminuire della dimensione verticale " $l$ " dalla lastra, fermi restando $x$, e $z$, e gli effetti $e$. $m$. permangono gli stessi.

Le coppie di profili $M, N$ offrono massimi all'origine: (v. foglio l, foglio 7), oppure: (fogli da 1 a 6 per $M$, da 7 a 11 per $N$ ). Le coppie dei profili $P$ e $Q$ hanno valori nulli all'origine del riferimento, con massimi che, per piccoli valori di $k$, danno modo di determinare la semiampiezza della lastra in quanto essi si pronunciano per valori di: $x / l=1$, per grandi valori di $k$ i massimi si spostano invece verso: $x / l>1$, con un limite superiore: $l<x_{\mathrm{m}}$ : (v. fogli 12 e seguenti fino a 17 per $P$, e da 18 fino a 22 per $Q$ ).

Alla coppia di profili simili $J_{1}, J_{1}^{\prime}$, relativi alle componenti verticali (max. all'origine), $c$. m. vorticoso nella lastra orizzontale, corrisponderà perciò la coppia dei profili simili $P, Q$ del $c$. $m$. vorticoso nella lastra verticale; e così a quella dei profili $J_{0}, J_{2}^{\prime}$ dellc componenti orizzontali nel c. e. m. s. nella lastra orizzontale (che s'annullano all'origine), corrisponderà la coppia dei profili $M, N$ del $c . e . m . s$. delli: lastra verticale.

Solo a causa delle posizioni diverse, ruolate di $90^{\circ}$, delle lastre conduttive piane, sedi di correnti vorticose, i profili delle componenti verticali nell'un caso si trasformano in profili delle componenti orizzontali nell'altro caso, e viceversa.

L'assunto posto ̀̀ così sufficientemente suffragato con questi esempi desunti da teorie rigorose, da studi inoltre su modelli e sul terreno. come potrò mostrare più esaurientemente tra qualche tempo, riordinando il materiale di una lunga sperimentazione modellometrica compiuta nel passato, a conforto della teoria data $\left({ }^{2}\right)$.

Analoghi diagrammi (ottenuti invece in precedenza con teorie schematiche, o approssimative), sia del gruppo $(M, N, P, Q$.) che del gruppo $\left(J_{1}, J_{1}^{\prime}, J_{2}, J_{2}^{\prime}\right)$, appaiono nelle opere di Heine, Sundberg. Hedstrom, ecc., come pure in diversi miei Studi $\left(^{6}\right)$, senonché le influenze e. $m$. $s$. dei cosidetti " concentratori spaziali di corrente" considerati, non possono avere che un valore puramente indicativo, data l'empiricità delle teorie stesse. 
Lin calcolo del genere (delle componenti orizzontale e verticale del $c . m$. s., in superficie) relativo ad un "indotto" sottile lastriforme, ad una certa profondita nel suolo, prisma sottile a sezione rettangolare, paralsolica, trapezoidale, $\infty^{\text {te }}$ o molto esteso, normalmente ad un prefissato piano verticale, con diversi angoli d'immersione (da zero gradi, orizzontalità, a novanta gradi, verticalitì), (clie qui non riporto per brevità), mostra l'evoluzione progressiva, in funzione dell'angolo d'immersione, dei rapporti di tali componenti di c. m. s., che, agli estremi s'invertono, come qui evidenziato.

Le torie classiche di Mayr, Levi-Civita, Pollaczek, Abraham, Haberlandt, con i numerosi sviluppi e applicazioni, riguardano, ¿̀ ben noto, " induttori " chiusi o aperti (indefiniti rettilinei, o di lunghezza finita), e "indotti ideali " nel suolo (orizzonti sottili, w $^{\text {te }}$ estesi), con risultati però che mantengono sempre un carattere indicativo (o qualitativol ai nostri fini (dovendosi considerare invece " indotti reali", di dimensioni perciò finite).

Le correnti vorticose, negli “ indotti ideali " e conseguenti $c$. $e$. m. s., (nel suolo o nell'aria), soddisfacenti all'equazione delle onde, ubbidiscono, in tali casi, a "condizioni limiti" solo limitatamente alla superficie di separazione aria-suolo (con riduzione, tra l'altro, del problema da tri a bidimensionale), e ad ulteriori condizioni di singolariti e di simmetria. Se viceversa l'u indotto", sia pure lastriforme, è di dimensioni finite, le condizioni limiti imposte dal problema aumentano ancora, con la conseguenza però che $i$ risultati, $-v \cdot\left({ }^{2}\right)-$ acquistano finalmente quel carattere concreto e quantitativo voluto, utilizzabile ai fini della prospezione e. $\mathrm{m}$. del sottosuolo.

Nei seguenti n. 22 fogli riporto n. 100 curve, relative alle influenze di $c . m . s . \bar{B}$, d'una lastra minerale, di dimensioni 21 lungo $z$, w lungo $x$, molto estesa lungo l'asse delle $y$, influenze calcolate per diverse situazioni della lastra, secondo le formule $(\stackrel{2}{)}$.

I diagrammi riportano i coefficienti $M, N, P, Q$, relativi alle formule che danno $B_{\mathrm{sqx}}, \bar{B}_{\mathrm{sqz}}$.

Questi coefficienti sono stati calcolati nelle situazioni seguenti: $l=1 ; 3 ; 5 ; 7 ; 10 \quad ; \quad k=z: l=2 ; 3 ; 5 ; 7 ; 10 ;$ per $h=x: l$ variabile fra 0,1 e 10.

In ascisse sono riportate costantemente le $h=x / /$ (scale logaritmiche) mentre in ordinate sono riportate le $\bar{B}_{\text {s }}$ (scale lineari).

Per rintracciare agevolmente le curve precede un apposito indice 
col numero del foglio e le scale di riferimento in funzione dei coefficienti $M, N, P, Q$, e di $l$ e $h$.

Ringrazio il mio Aiuto Dott. Renato Trudu per la sua collaborazione nella redazione dell'annesso Album.

Cagliari - Istituto di Geofisica Mineraria - Gennaio 1953.

\section{RIASSUNTO}

L'A. riassume brevemente dapprima i suoi Studi sui campi clettromagnetici vorticosi in lastre infinitamente estese $e$ in lastre di dimensioni finite, con la soluzione del cosidetto problema della «lastra finita» conduttiva.

Rilevata la sostanziale differenza analitica tra $i$ due problemi concernenti tali tipi di "indotti", ideali e reali, $l$ ' $A$. ne evidenzia le analogie delle soluzioni, con comparazione dei conseguenti diagrammi.

$L^{\prime}$ 'A. si sofferma infine a giustificare qualche apparente discrepanza, relativa all'influenza dell'orientamento della lastra conduttiva rispetto alla superficie orizzontale del suolo.

Dà infine la possibilità di poter "catalogare" tali influenze e.m.s. di campi vorticosi, qualunque siano gli emittori, le ubicazioni e gli orientamenti dei giacimenti conduttivi lastriformi.

Completa la Nota con un Album d'influenze e. m. di lastre minerali conduttive.

\section{SC MIMARY}

The Author briefly summarizes his studies on electromagnetic field vortices in infinitely extended planes and in planes of finite dimensions, with the solution of the so-called problem of the conducting "finite plane".

The substantial analytical difference between the tuo problems concerning such "inductions", ideal and real, is revealed and the author shous analogies of the solutions, and a omparison of the consequent diagrams.

The author pauses at the end to justify some apparent discrepancies relative to the influence of the orientation of the conducting plane to the horizzontal surface of the soil. 
The conclusion is concerned with the possibility of being able to catalogue the influences of such electromagnetic field vortices. whatever the sources are, and the positions and orientations of the plane conducting layers.

\section{BIBLIOGRAFIA}

(1) A. Belluigr: L'impiego della Geofisica Applicata in Miniera. Experientia, vol. V/3, 1949, pag. III, Basel.

(2) A. Belluigi: The Elestromagnetic Field Due To Induced Currents in a conductive Slab of Finite Dimensions. Geophysics, 1950.

(3) A. Belluigr: Il campo e.m. dovuto a correnti indotte in lastre conduttive di dimensioni finite o estese. Pubbl. della Facoltà d'Ingegneria dell'Università di Cagliari, 1950.

(4) A. Belluigr: Problemi fondamentali concernenti Limpiego dei m. induttivi in Miniera. Rend. Seminario Facoltà di Scienze della Università di Cagliari, 1948.

(5) A. Belluigr: Su alcuni orientamenti teorici per le misure e.m. in galleria di Miniera. Rivista di Geofisica Applicata, Milano 1950.

(6) A. Bellugr: Sui campi elettromagnetici a bassa frequenza. Rivisti di Geofisica Applicata, Milano 1952.

(7) A. Belluig1: Sulle interpretazioni delle influenze e.m. di giacimenti minerali metallici eccitati con c.a. Pubblicazioni della Facolti di Ingegneria della Università di Cagliari, n. 1, 1950. 


\section{INDICE DELL'ALBUM}

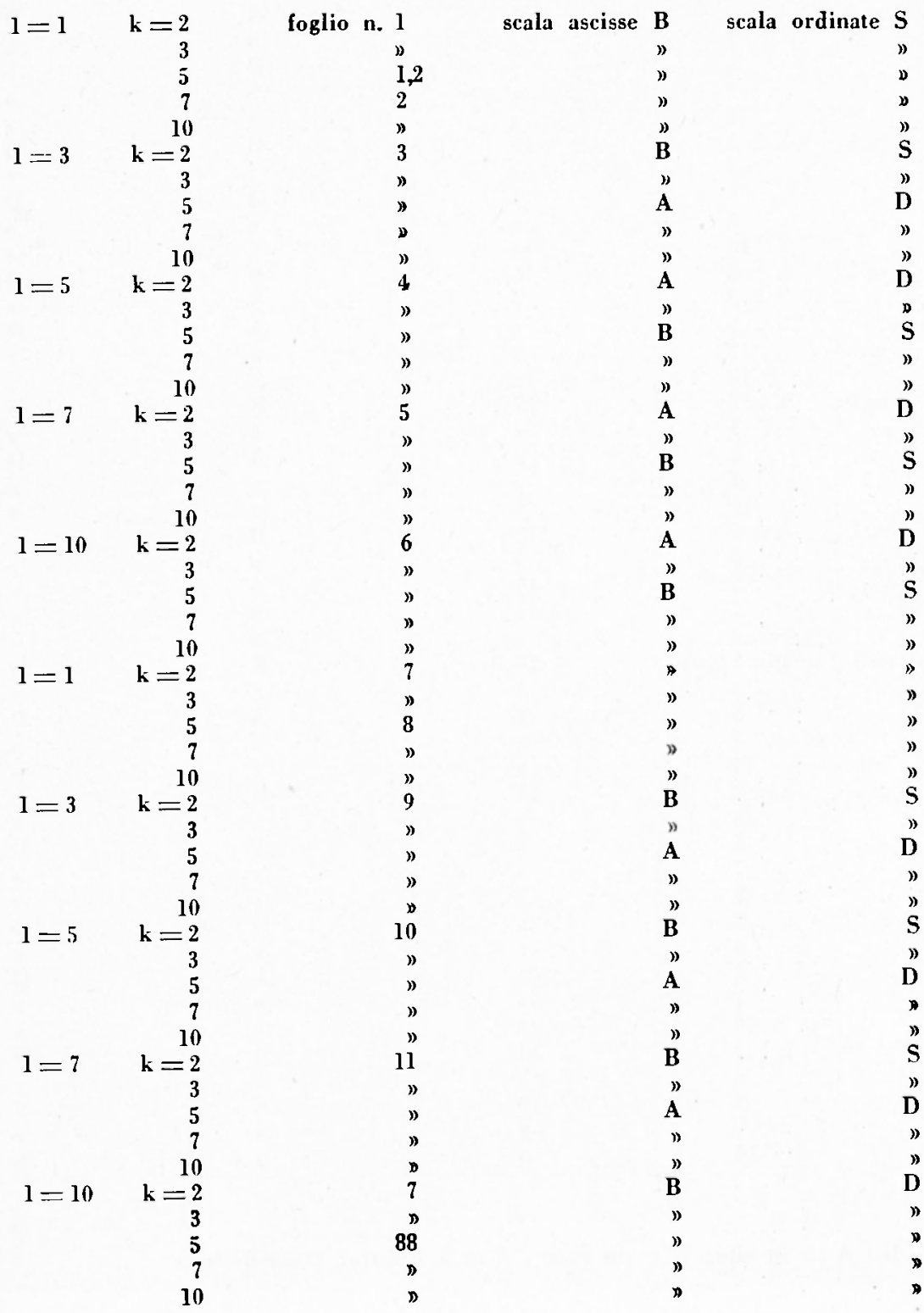

N. $B .: A=$ in alto; $B=$ in basso; $S=$ a sinistra; $D=$ destra. 


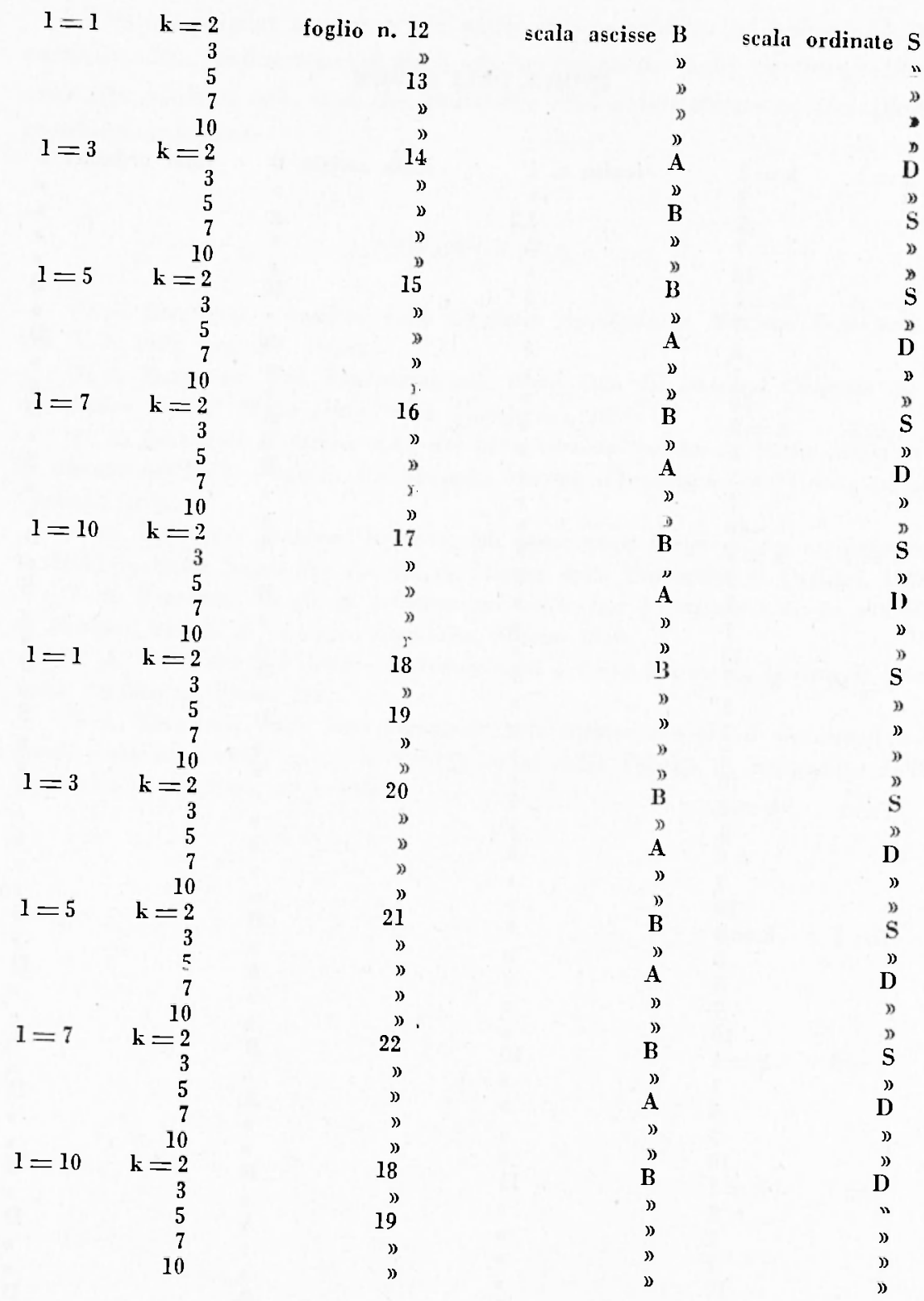

N. B.: $A=$ in alto; $B=$ in basso; $S=$ a sinistra; $D=$ destra. 


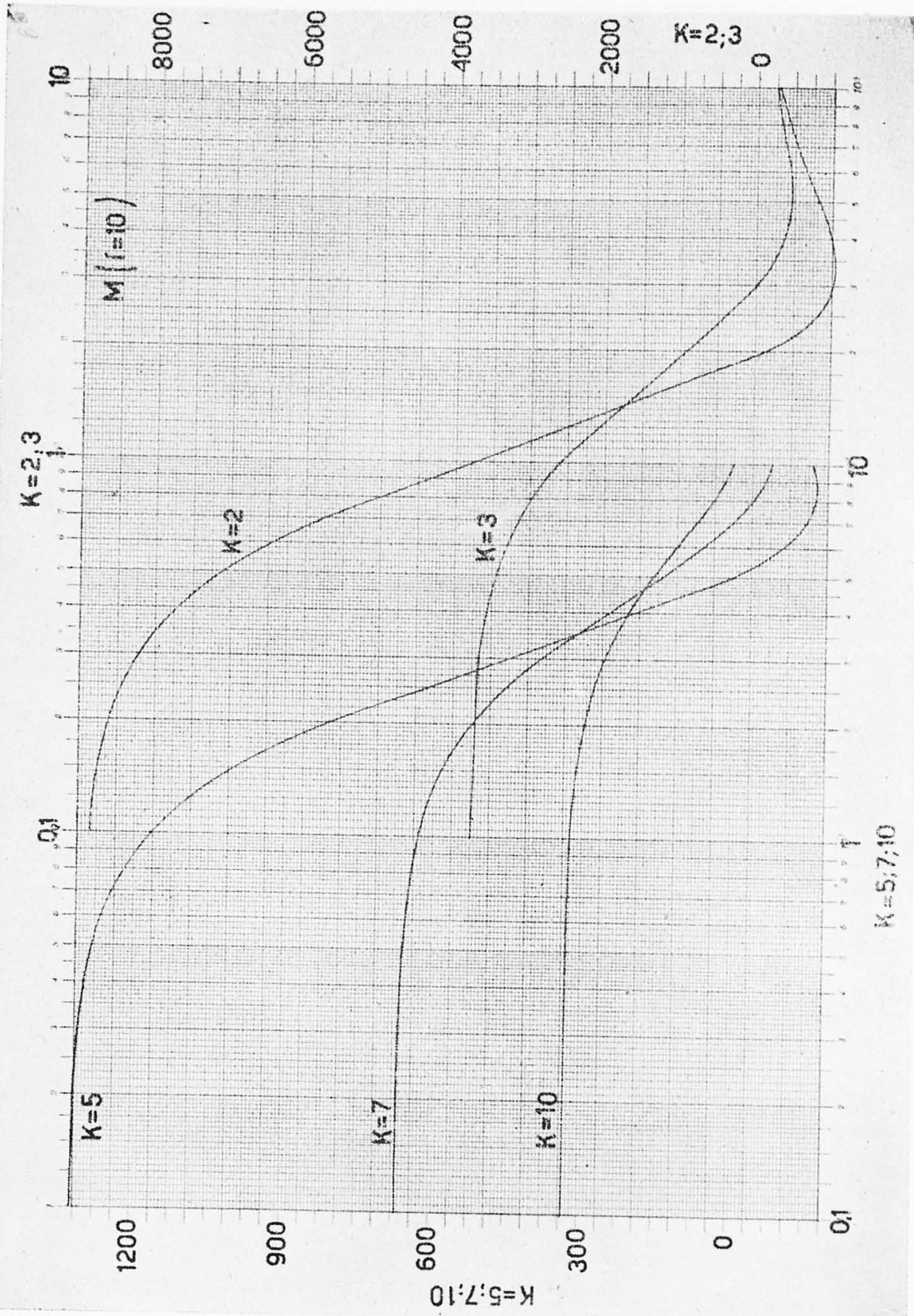




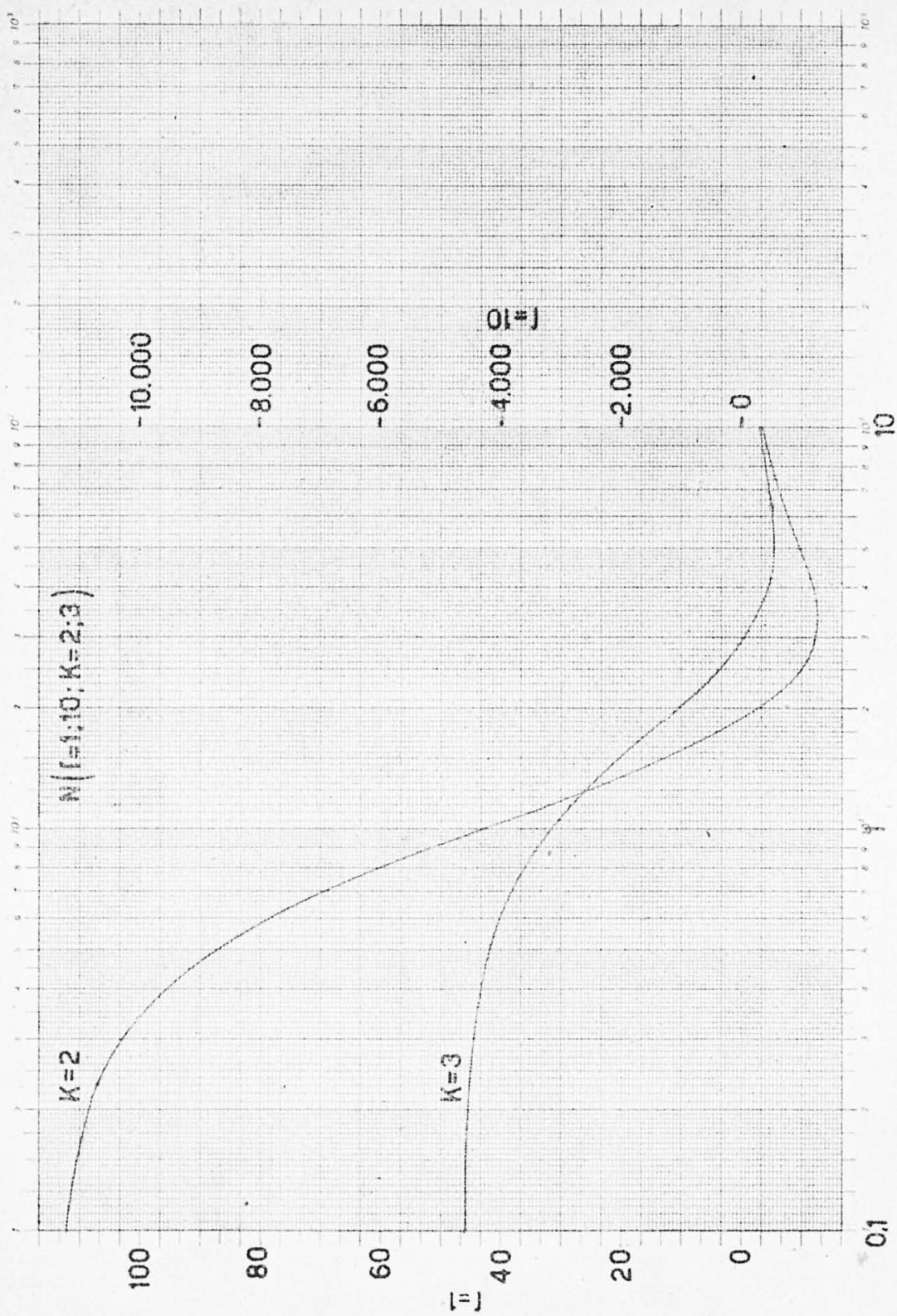




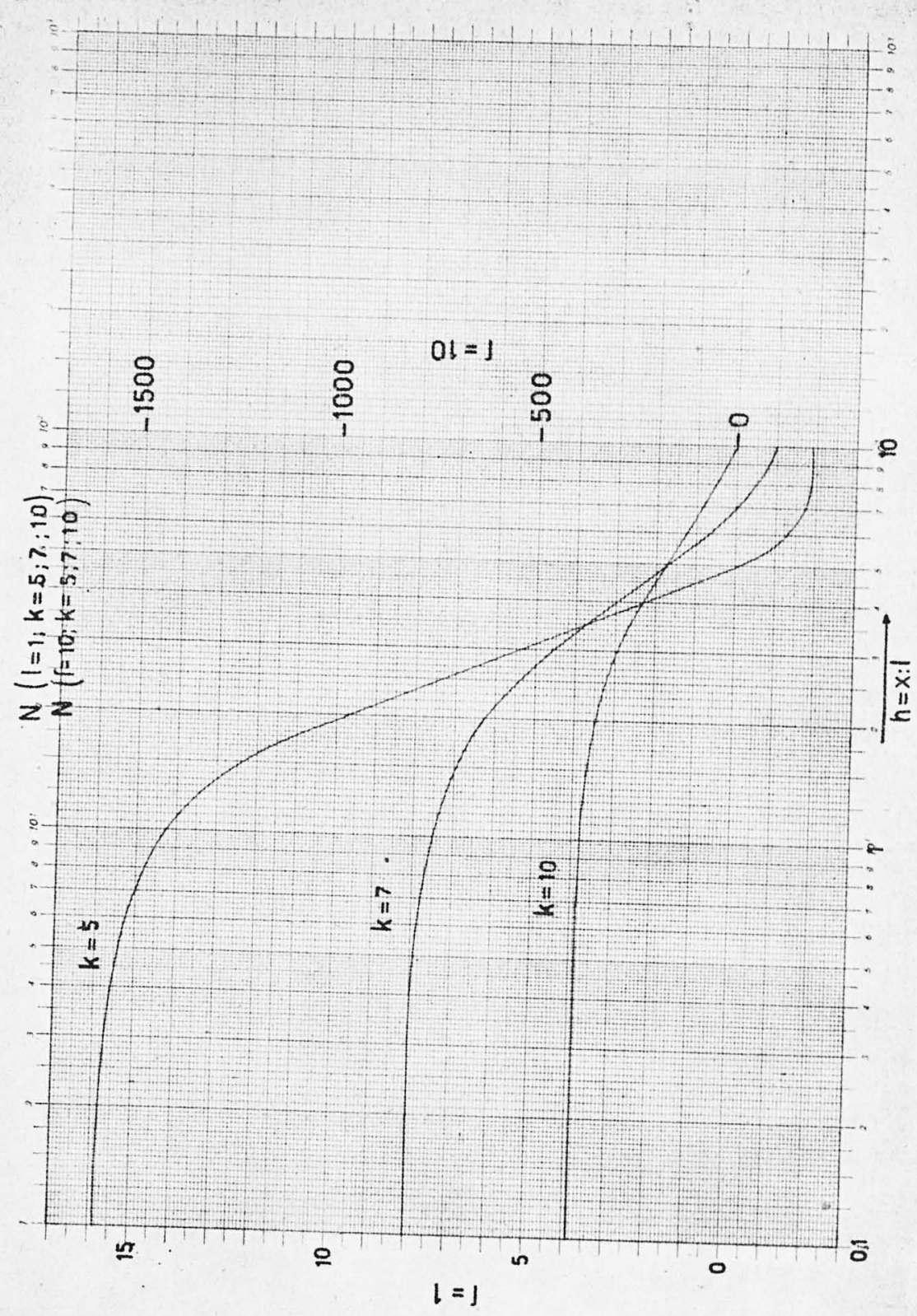


CAMPI ELETTROMAGNETICI VORTICOSI IN LASTRE INFINITAMENTE ESTESE

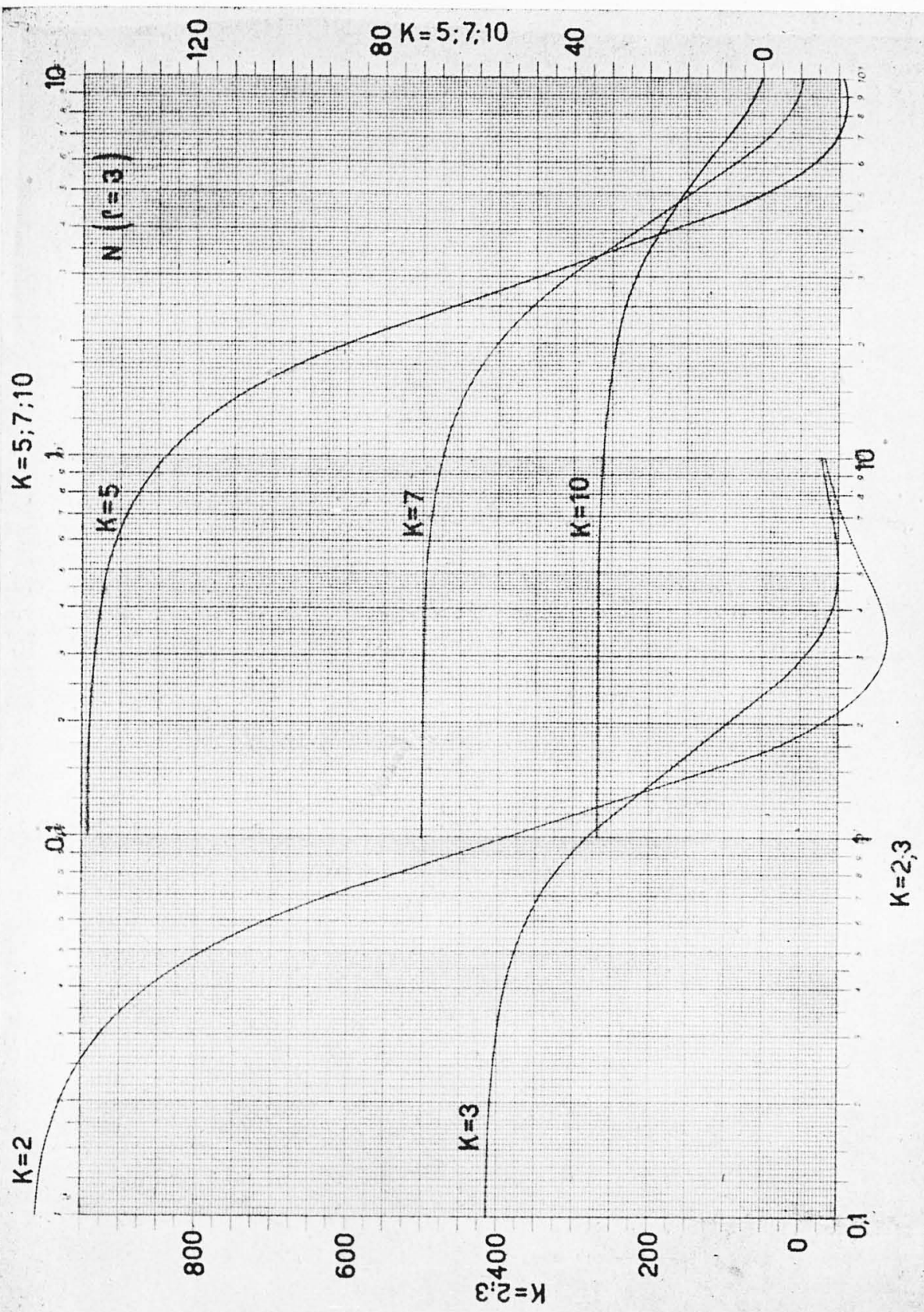


A. BELLUIGI

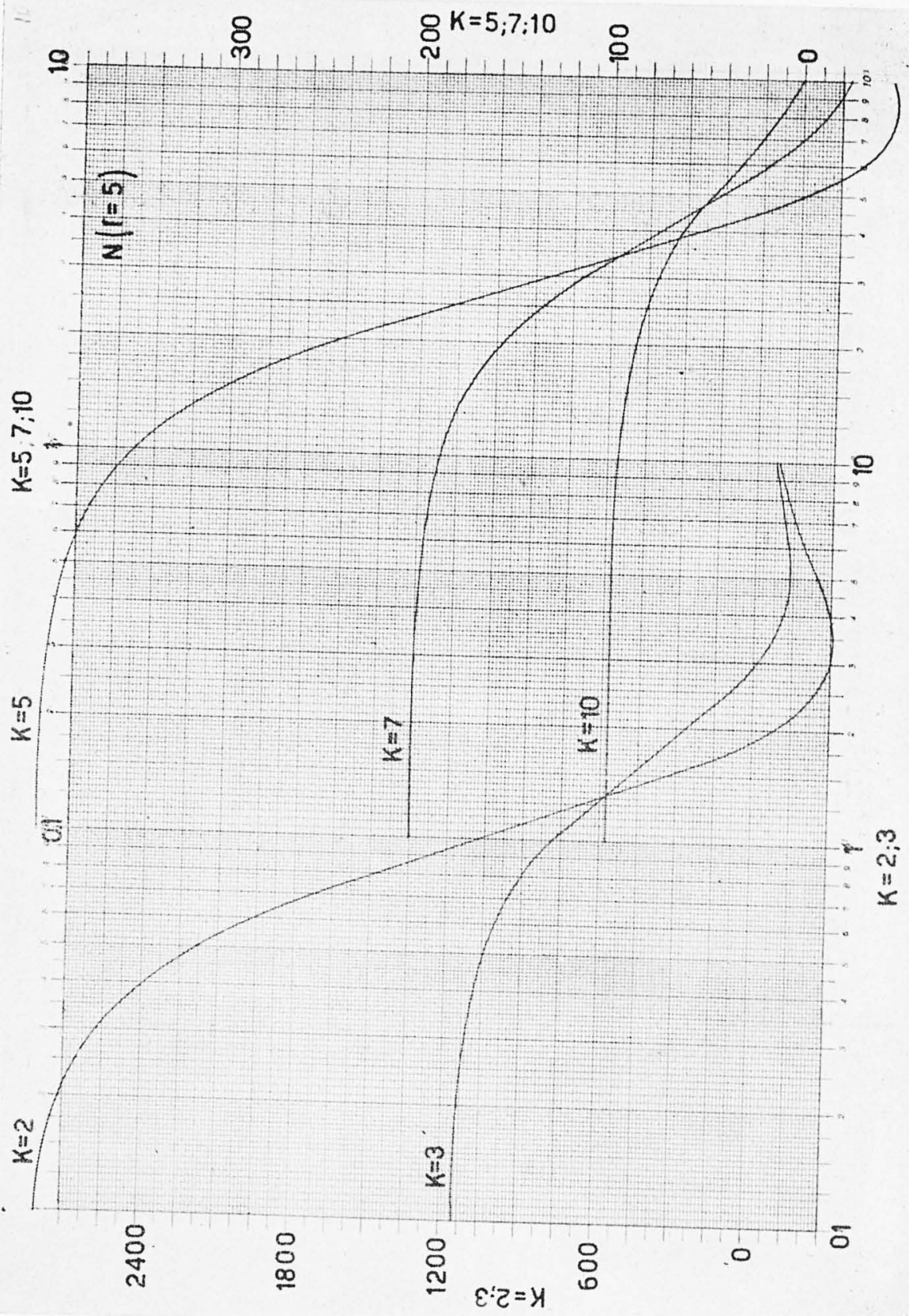




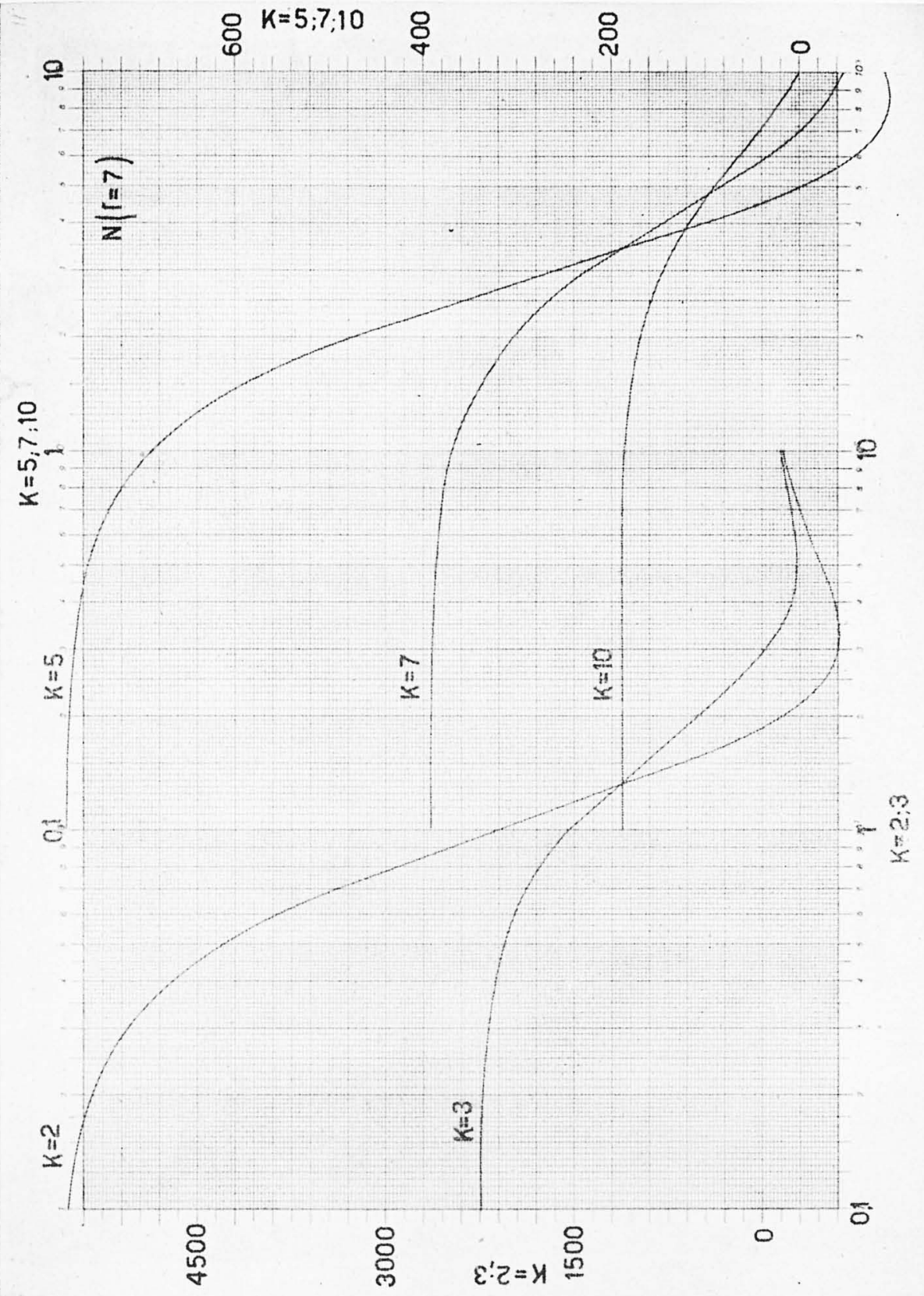




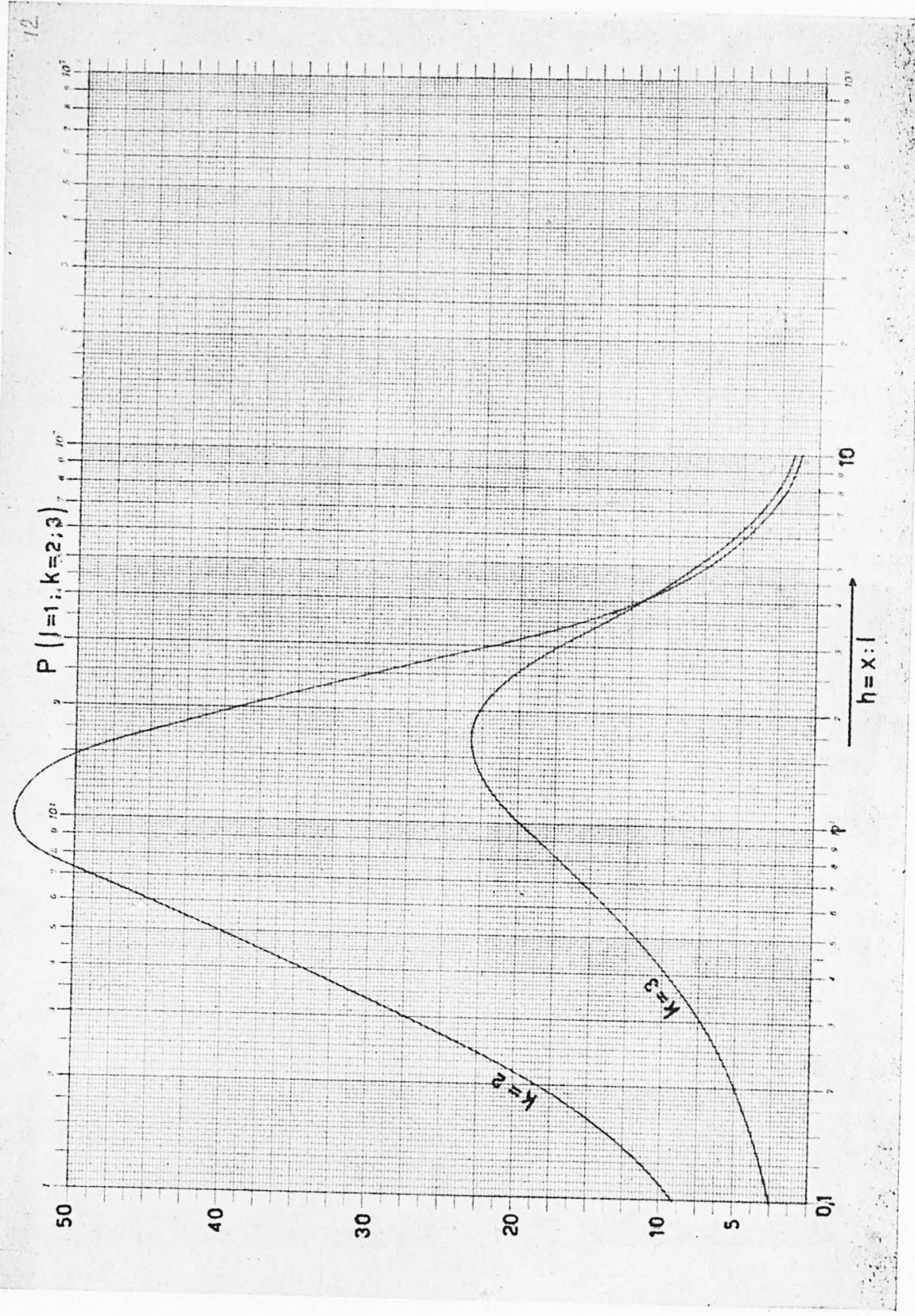




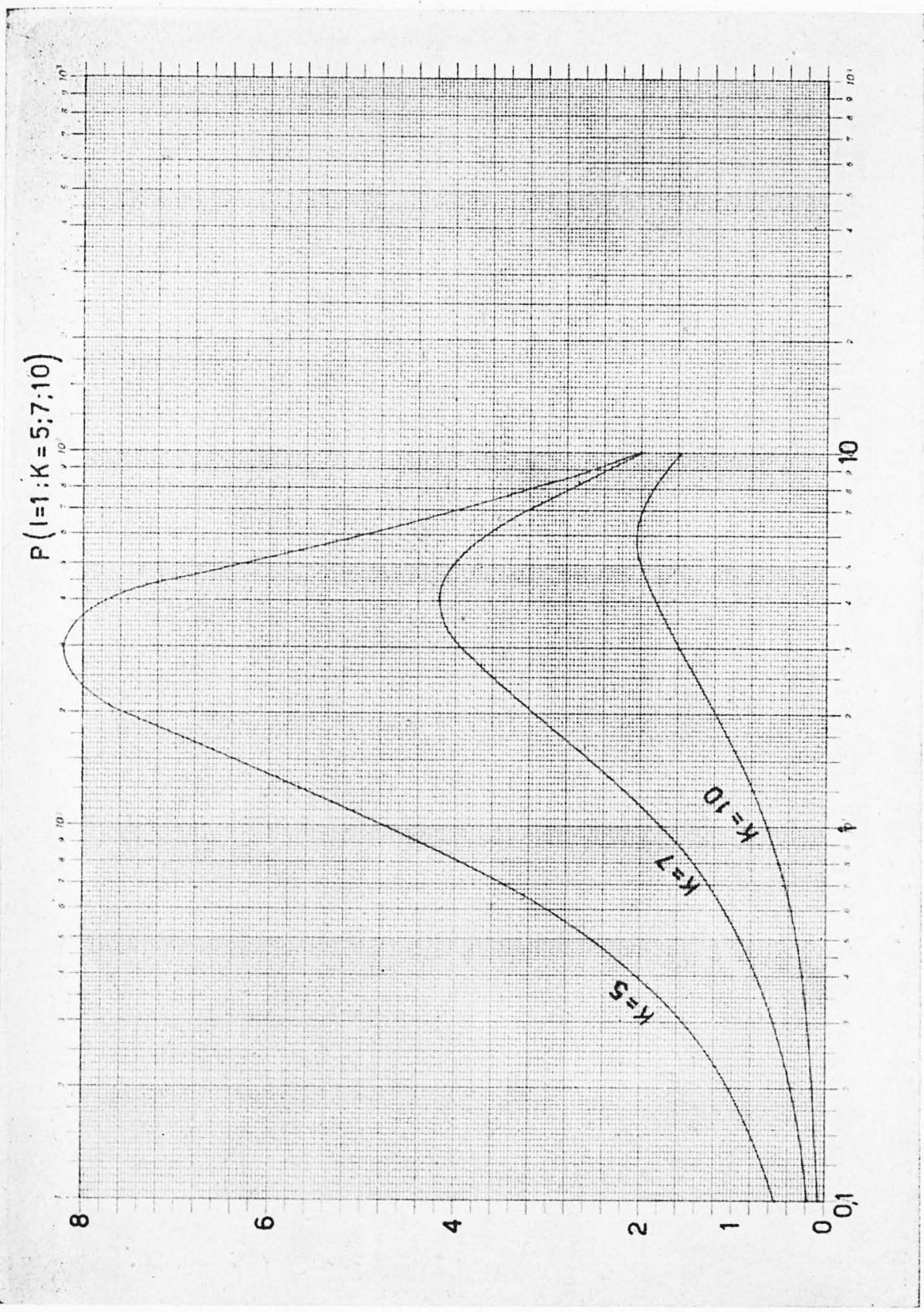


A. BELLUigI

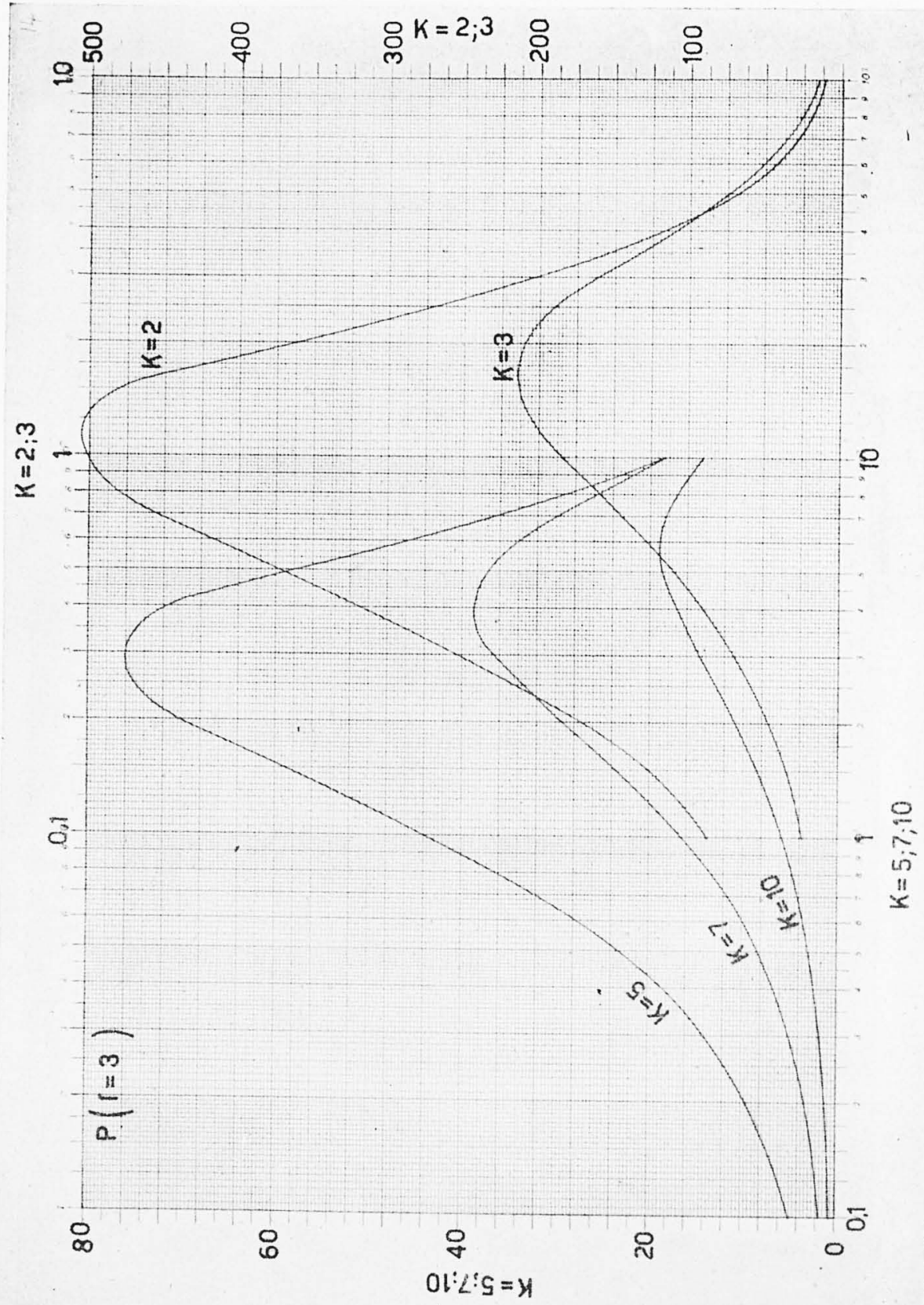


CAMPI ELETTROMAGNETICI VORTICOSI IN LASTRE INFINITAMENTE ESTESE

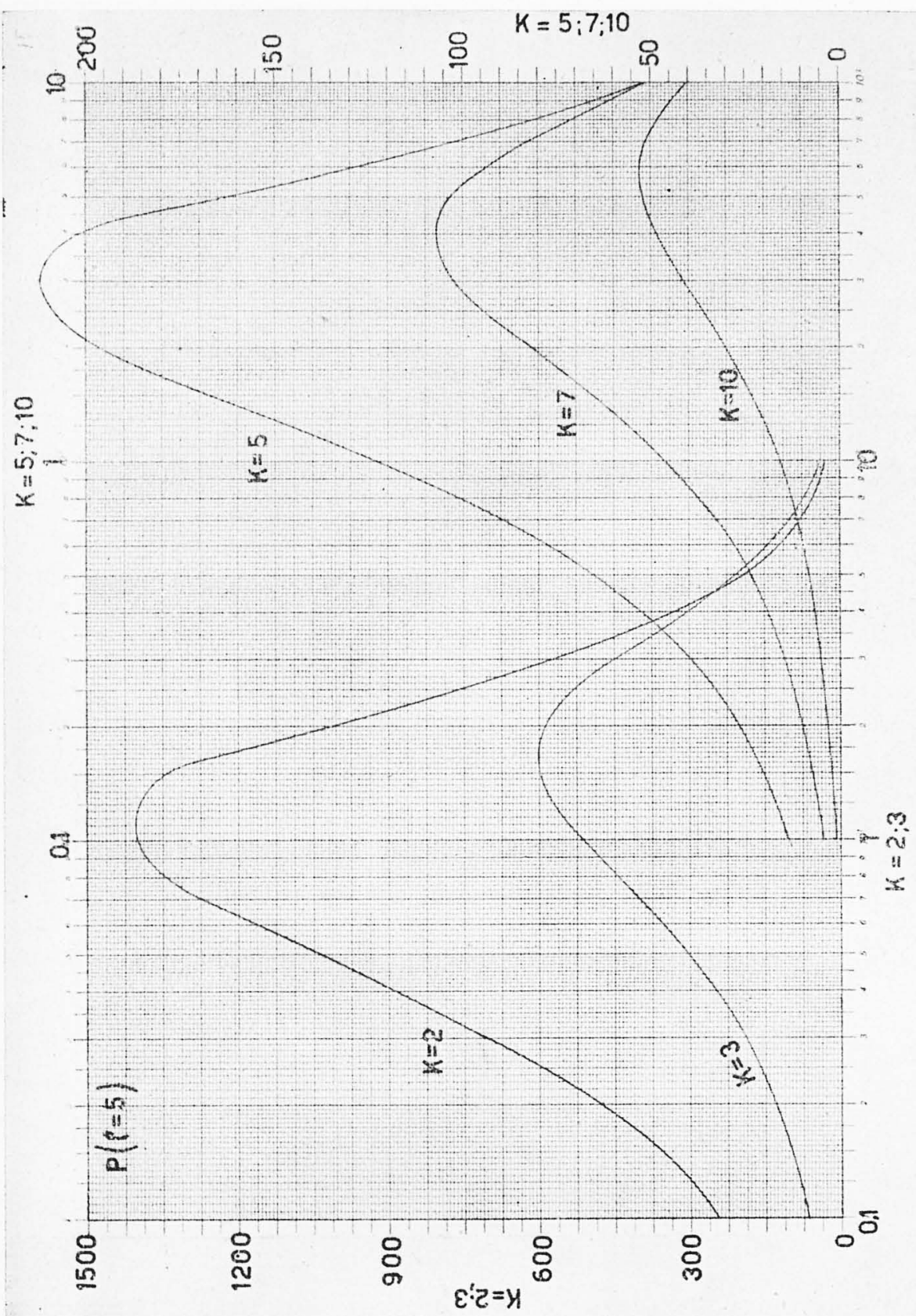


A. Belluigi

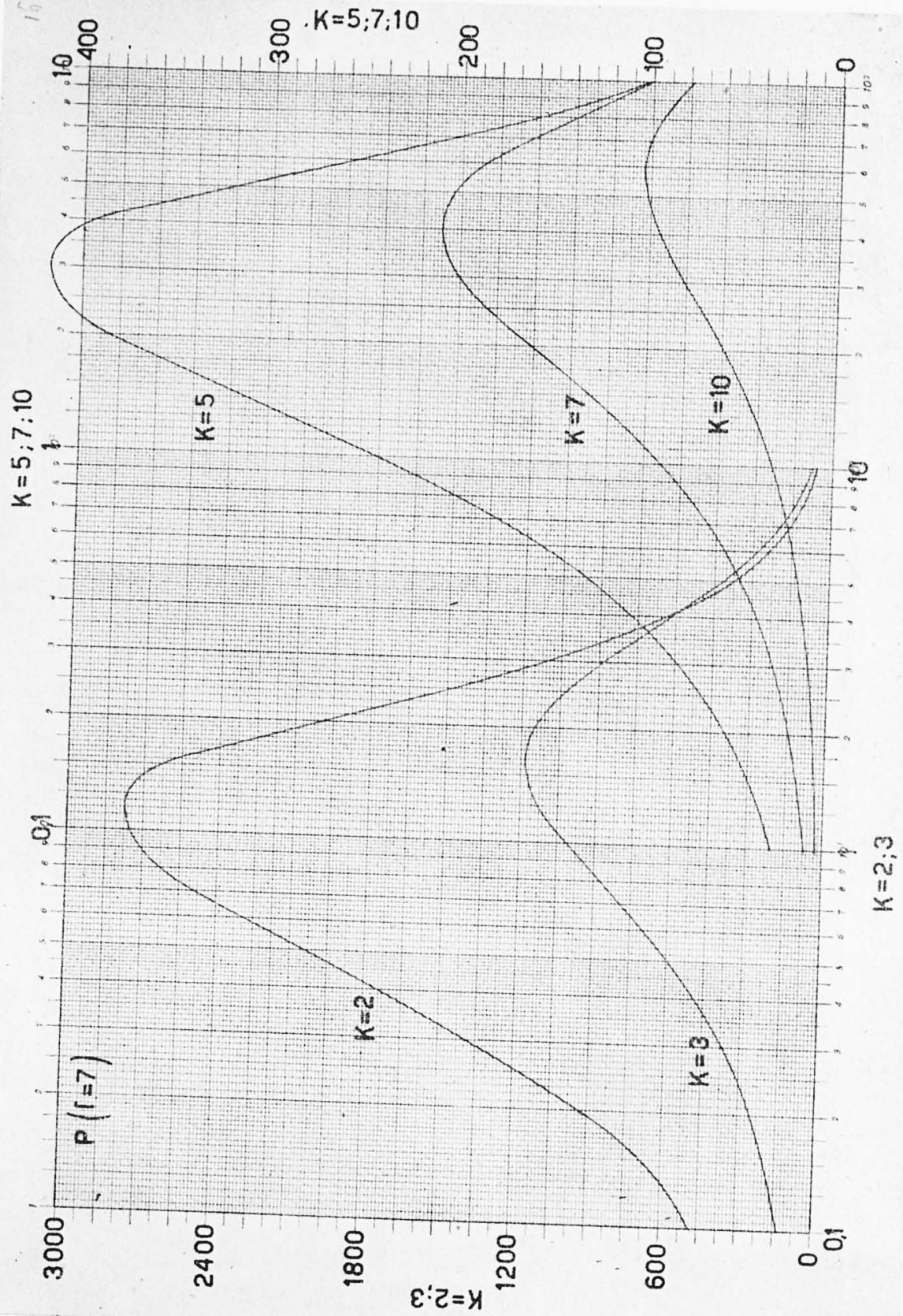




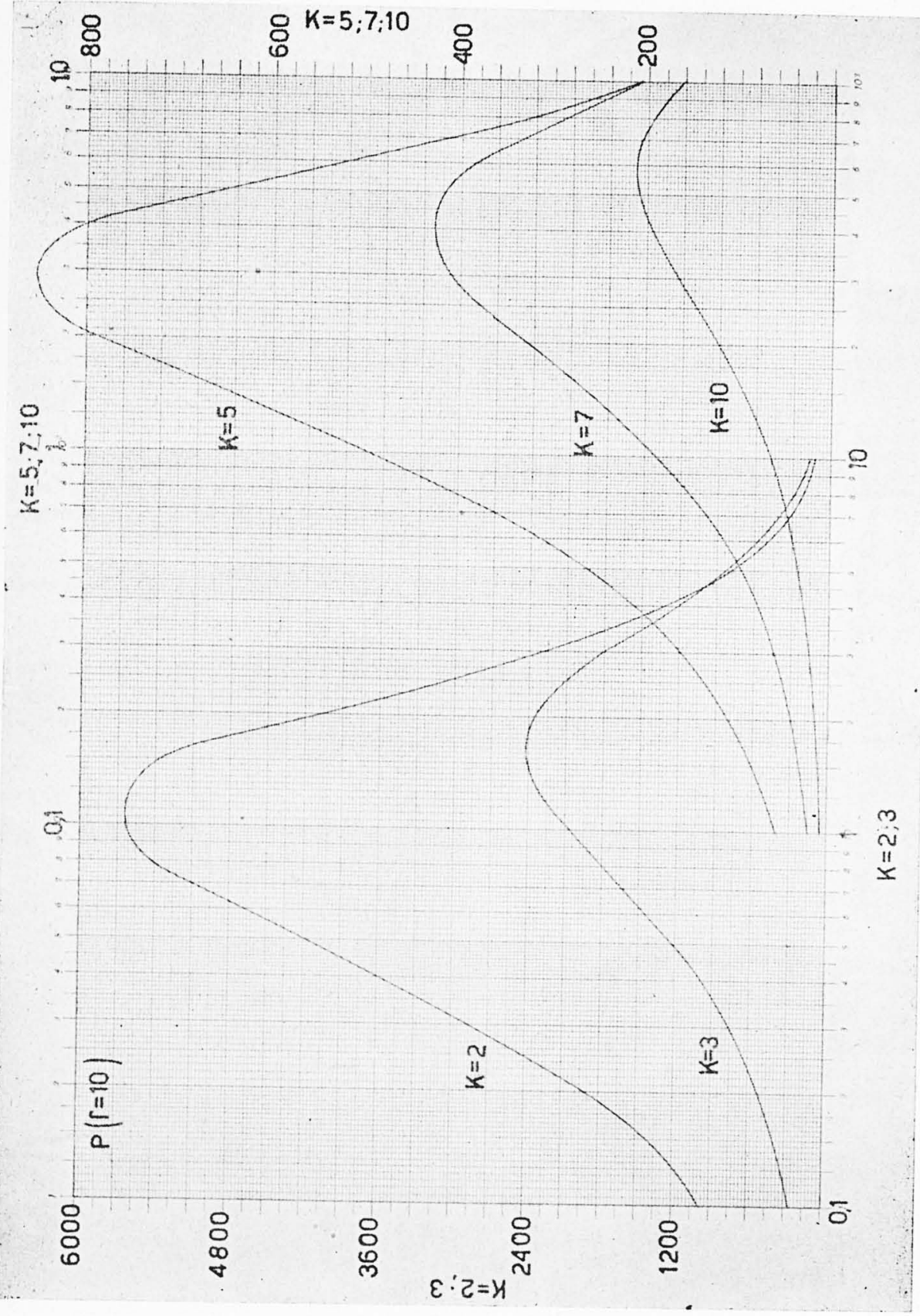


A. Belluigi

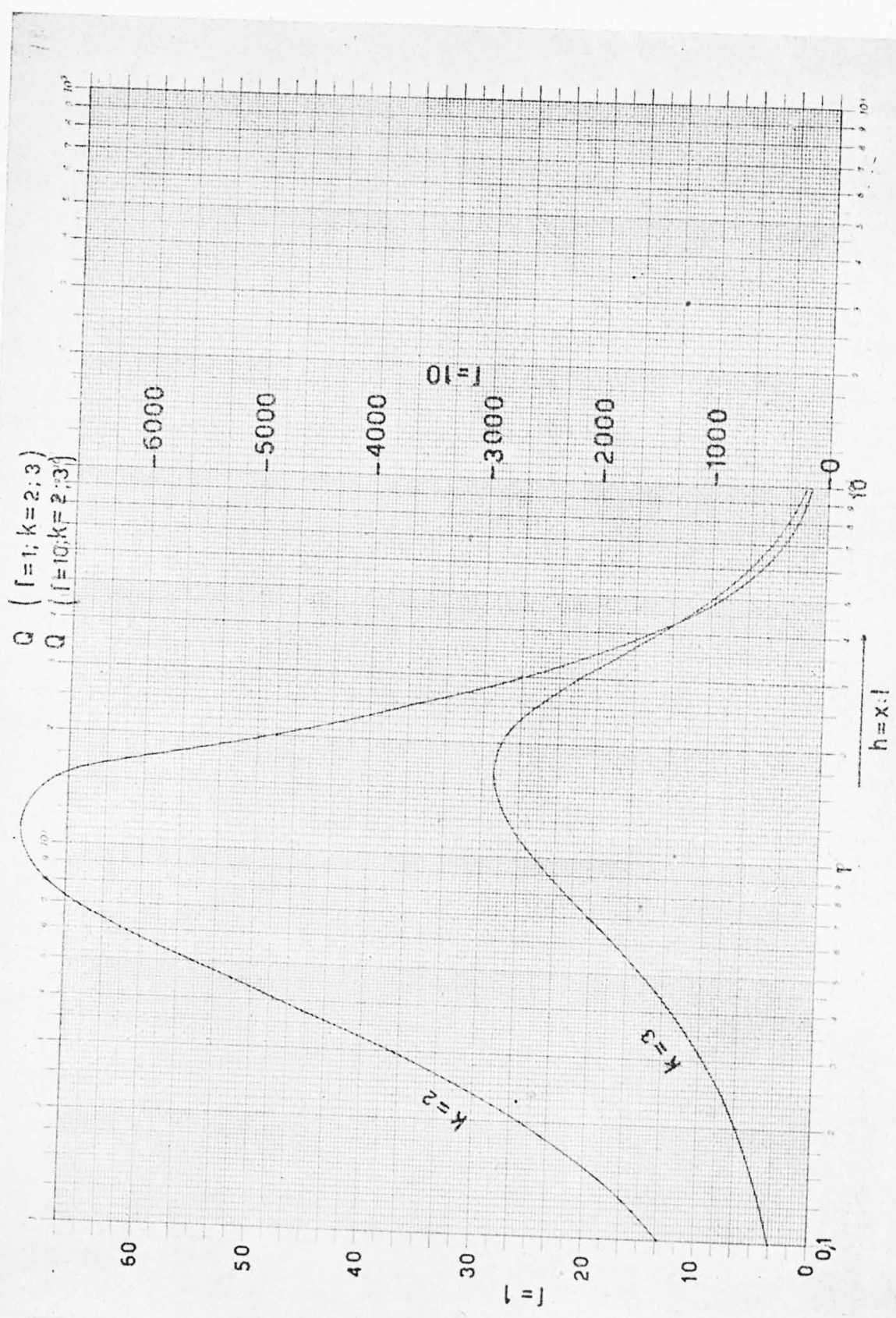




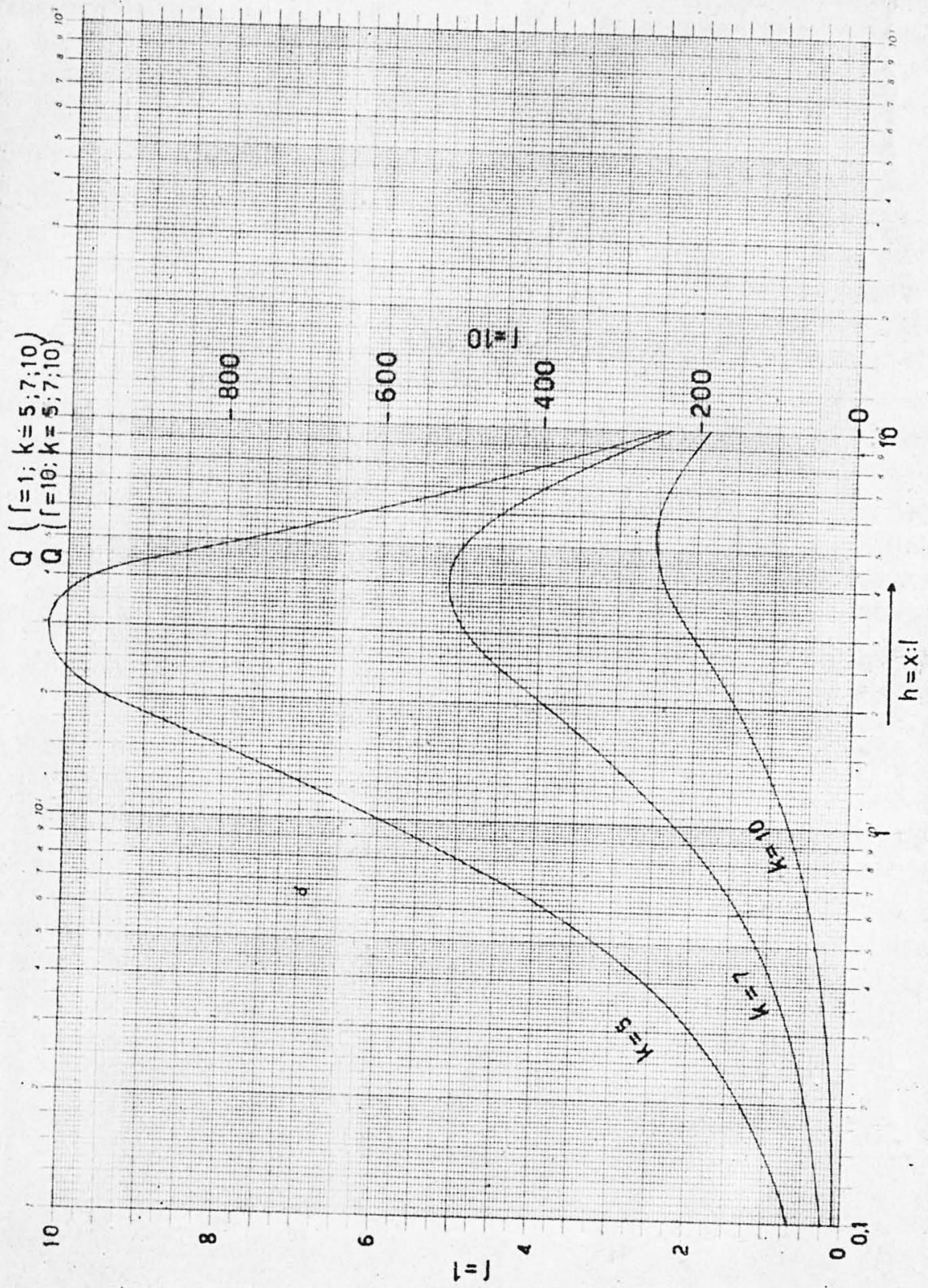


282

A. BELLUIGI

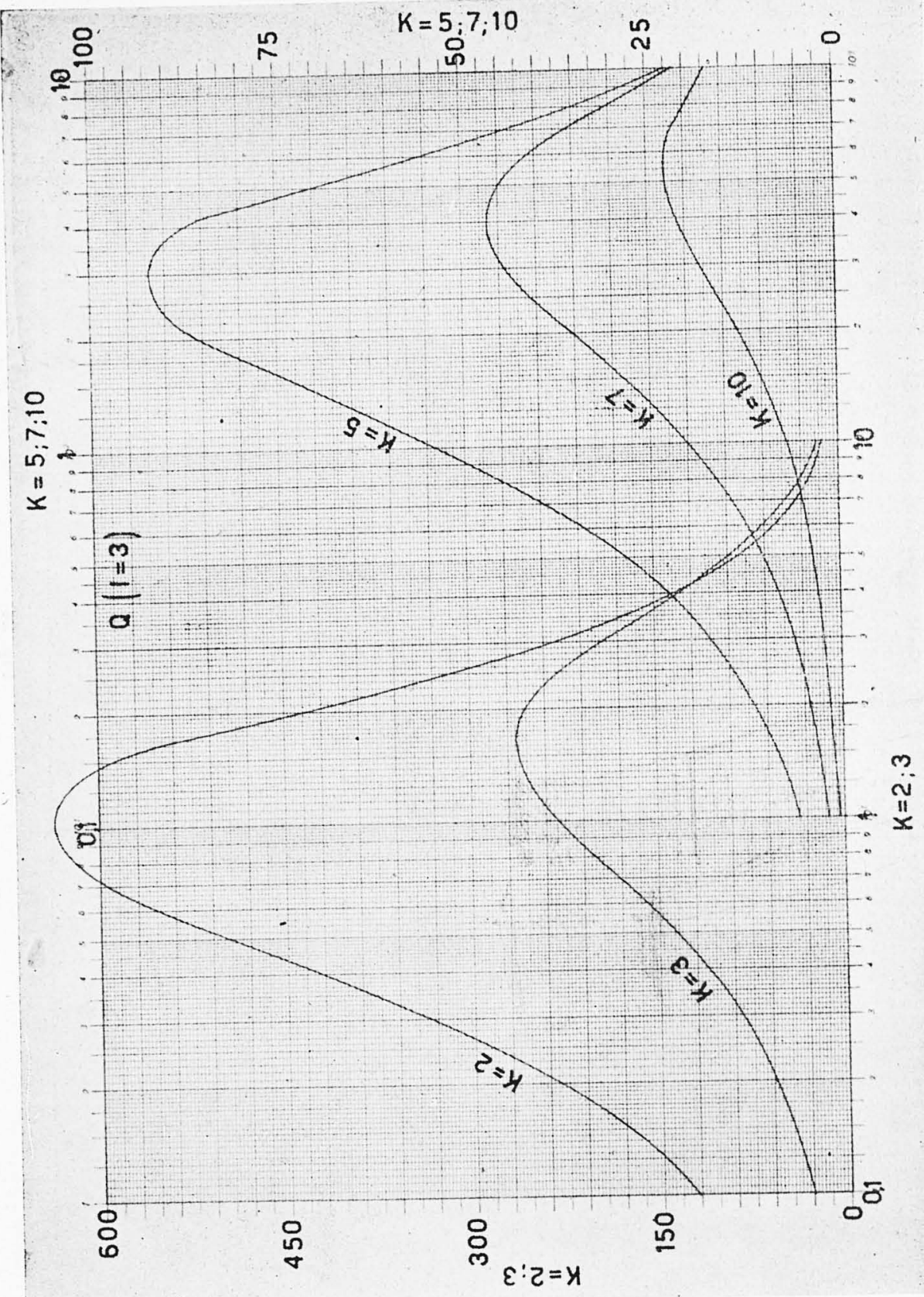




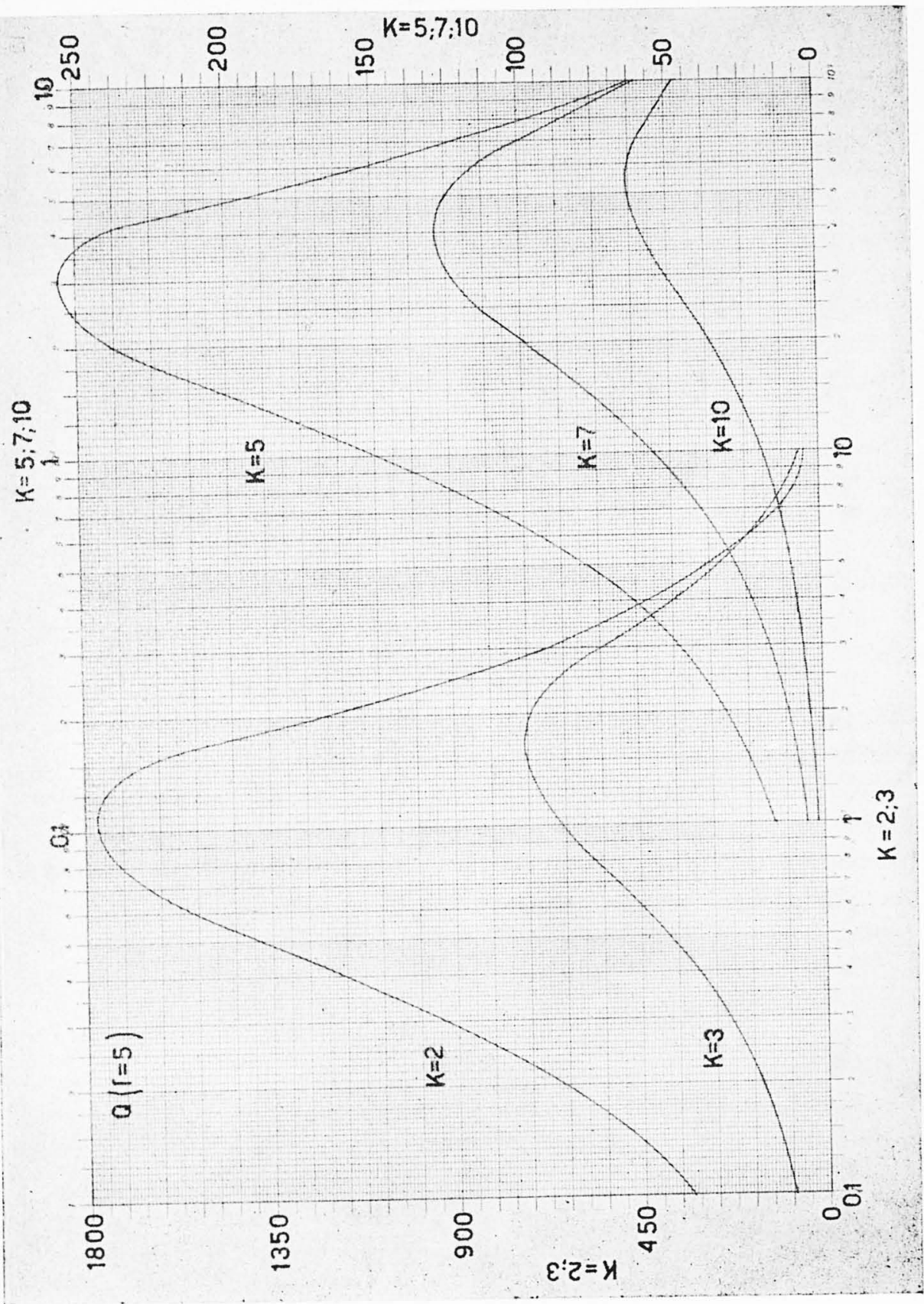




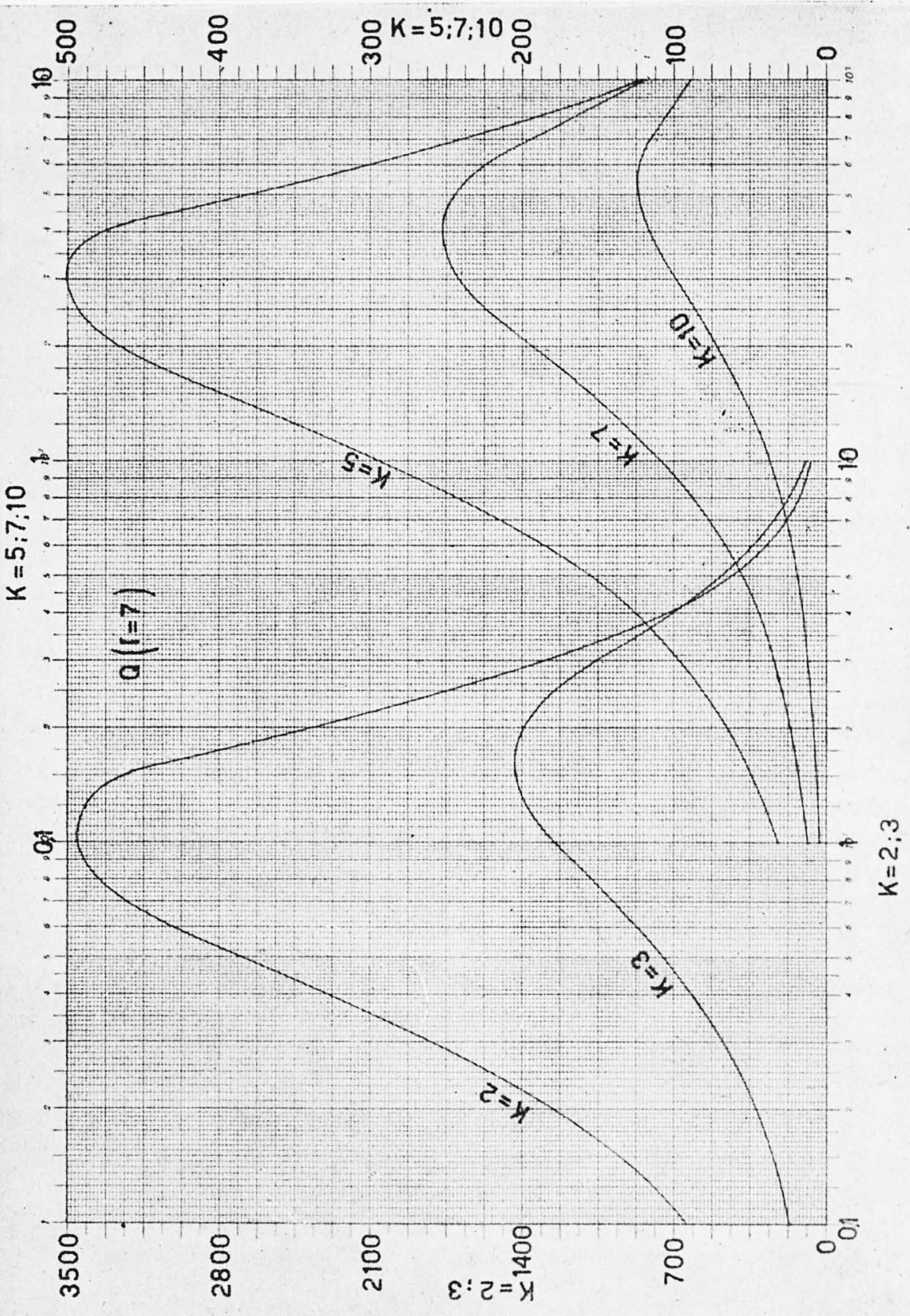

